\title{
STATIONARY BLACK HOLES IN A GENERALIZED THREE-DIMENSIONAL THEORY OF GRAVITY
}

\author{
Paulo M. Sá \\ Sector de Física, Unidade de Ciências Exactas e Humanas, Universidade do Algarve, \\ Campus de Gambelas, 8000 Faro, Portugal. \\ José P. S. Lemos \\ Departamento de Astrofísica, Observatório Nacional-CNPq, \\ Rua General José Cristino 77, 20921 Rio de Janeiro, Brasil, \\ $\&$ \\ Departamento de Física, Instituto Superior Técnico, \\ Av. Rovisco Pais 1, 1096 Lisboa, Portugal.
}

\begin{abstract}
We consider a generalized three-dimensional theory of gravity which is specified by two fields, the graviton and the dilaton, and one parameter. This theory contains, as particular cases, three-dimensional General Relativity and three-dimensional String Theory. Stationary black hole solutions are generated from the static ones using a simple coordinate transformation. The stationary black holes solutions thus obtained are locally equivalent to the corresponding static ones, but globally distinct. The mass and angular momentum of the stationary black hole solutions are computed using an extension of the Regge and Teitelboim formalism. The causal structure of the black holes is described.
\end{abstract}




\section{Introduction}

A few years ago, Bañados, Teitelboim and Zanelli (BTZ) showed that three-dimensional General Relativity with a negative cosmological constant admits a black hole [目]. This solution exhibits many of the characteristics of four dimensional black holes: it arises from the gravitational collapse of matter [2], it is a thermodynamic object with a well defined temperature and entropy [3] and it can be matched to a three-dimensional perfect fluid star [ [⿴囗十). Furthermore, the BTZ black hole can be transformed into a four-dimensional black string in General Relativity [5] (for a review see [6, 7]).

A interesting aspect of the BTZ black hole lies in its relation with string theory. This black hole was shown to be also a solution to low energy string theory with a non-vanishing antisymmetric tensor [8, 9]. Under duality it is equivalent [8] to a black string [10] which, in the non-rotating case, is simply the three-dimensional black hole obtained by taking the product of the two-dimensional string black hole [11, 12] with $S^{1}$. It was further shown by Kaloper [9] that the BTZ black hole is also a solution of topologically massive gravity. Thus, the BTZ black hole is a solution of alternative theories of gravity. That fact has attracted considerable interest to the study of black objects in three-dimensional theories of gravity and led to some generalizations of the BTZ black hole.

Of particular interest are the generalizations of the BTZ black hole which are achieved by the introduction of a dilaton field [13, 14]. One such generalization can be acomplished by considering a action of the Brans-Dicke type,

$$
S=\frac{1}{2 \pi} \int d^{3} x \sqrt{-g} e^{-2 \phi}\left[R-4 \omega(\partial \phi)^{2}+4 \lambda^{2}\right],
$$

where $g$ is the determinant of the three-dimensional metric, $R$ is the curvature scalar, $\phi$ is a scalar field, $\lambda$ is a constant and $\omega$ is the three-dimensional Brans-Dicke parameter. Each different $\omega$ yields a different dilaton gravity theory; $\omega=-1$ corresponds to the low energy string action with vanishing antisymmetric tensor field, $\omega=0$ corresponds to a theory related to fourdimensional General Relativity with one Killing vector and for $\omega= \pm \infty$ one obtains three-dimensional General Relativity. Despite its simplicity, the dilaton gravity theory given by the above action admits a very rich structure of black hole solutions.

In a previous work [14, we have found and analysed, for the full range of 
values of the parameter $\omega$, the static black hole solutions obtained from (11) with the following ansatz for the metric:

$$
d s^{2}=-e^{2 \nu(r)} d t^{2}+e^{-2 \nu(r)} d r^{2}+r^{2} d \varphi^{2}, \quad 0 \leq \varphi \leq 2 \pi .
$$

These black hole solutions have the form:

$$
\begin{gathered}
d s^{2}=-\left[a^{2} r^{2}-\frac{b}{(a r)^{\frac{1}{\omega+1}}}\right] d t^{2}+\frac{d r^{2}}{a^{2} r^{2}-\frac{b}{(a r)^{\frac{1}{\omega+1}}}}+r^{2} d \varphi^{2}, \\
\omega \neq-\frac{3}{2},-1, \\
d s^{2}=4 \lambda^{2} r^{2} \ln (b r) d t^{2}-\frac{d r^{2}}{4 \lambda^{2} r^{2} \ln (b r)}+r^{2} d \varphi^{2}, \\
\omega=-\frac{3}{2},
\end{gathered}
$$

with the dilaton field given by:

$$
e^{-2 \phi}=(a r)^{\frac{1}{\omega+1}}, \quad \omega \neq-1
$$

The constant of integration $b$ is positive and related to the mass of the black holes,

$$
\begin{array}{ll}
M=\frac{\omega+2}{\omega+1} b, & \omega \neq-\frac{3}{2},-1, \\
M=-4 \lambda^{2} \ln (b), & \omega=-\frac{3}{2} ;
\end{array}
$$

the constant $a$ is related to $\lambda$ and $\omega$.

For $\omega=-1$, which corresponds to the low energy three-dimensional string theory with a vanishing antisymmetric tensor field, the metric gives simply the three-dimensional Minkowski spacetime and the dilaton is constant. Thus, in what follows the case $\omega=-1$ will not be considered.

The causal structure, the geodesic motion of null and timelike particles in the black hole geometries and the ADM masses of the black hole solutions (3)-(5) were analysed in detail in ref. 14.

Recently, Cadoni [15] has analysed, from the point of view of duality symmetries, the theory given by action (1) and recover, in particular, the static solution (3). 
The purpose of this paper is to generalize the static black hole solutions (3)-(5) in order to include rotation.

\section{Stationary Black Hole Solutions}

Stationary black hole solutions of action (1) can be generated from the static black hole solutions by a simple coordinate transformation:

$$
\begin{aligned}
t & \rightarrow \beta t-\frac{\theta}{a^{2}} \varphi \\
\varphi & \rightarrow \beta \varphi-\theta t,
\end{aligned}
$$

where $\beta, \theta$ and $a$ are constants. The stationary black hole solutions thus obtained are locally equivalent to the corresponding static ones, but globally distinct for each value of the parameter $\theta$ [16].

Applying the above coordinate transformation to solutions (3) and (4) one obtains the following stationary black holes (for $\omega=-\frac{3}{2}$ we make $a^{2}=\lambda^{2}$ ):

$$
\begin{aligned}
& d s^{2}=-\left[\left(\beta^{2}-\frac{\theta^{2}}{a^{2}}\right) a^{2} r^{2}-\frac{\beta^{2} b}{(a r)^{\frac{1}{\omega+1}}}\right] d t^{2}-\frac{\beta \theta}{a^{2}} \frac{b}{(a r)^{\frac{1}{\omega+1}}} 2 d \varphi d t \\
&+\frac{d r^{2}}{a^{2} r^{2}-\frac{b}{(a r)^{\frac{1}{\omega+1}}}}+\left[\left(\beta^{2}-\frac{\theta^{2}}{a^{2}}\right) r^{2}+\frac{\theta^{2}}{a^{4}} \frac{b}{(a r)^{\frac{1}{\omega+1}}}\right] d \varphi^{2}, \\
& \omega \neq-\frac{3}{2},-1, \\
& d s^{2}= {\left[4 \lambda^{2} \beta^{2} r^{2} \ln (b r)+\theta^{2} r^{2}\right] d t^{2}-\left[4 \beta \theta r^{2} \ln (b r)+\beta \theta r^{2}\right] 2 d \varphi d t } \\
&-\frac{d r^{2}}{4 \lambda^{2} r^{2} \ln (b r)}+\left[\frac{4 \theta^{2}}{\lambda^{2}} r^{2} \ln (b r)+\beta^{2} r^{2}\right] d \varphi^{2}, \\
& \omega=-\frac{3}{2} .
\end{aligned}
$$

The dilaton field is given by eq. (5).

For the case $\omega \neq-\frac{3}{2},-1$, in order to have the standard form of the antide Sitter spacetime at spatial infinity we choose $\beta^{2}=1+\frac{\theta^{2}}{a^{2}}$. For $\omega=-\frac{3}{2}$ we choose $\beta=1$. 
The $\omega=0$ stationary black hole was discussed in ref. [17], where the three-dimensional gravity theory was obtained through dimensional reduction from four-dimensional General Relativity with one Killing vector field. This solution was further generalized to include charge [18]. Some of these black hole solutions were also analysed in ref. [19]; the action considered there differs from our action by a conformal transformation $g_{i j} \rightarrow e^{-4 \phi} g_{i j}$.

To define mass and angular momentum for the black hole solutions (10)(11) we apply the formalism of Regge and Teitelboim [20] (see also [14, 17]).

We write the stationary metric in the canonical form,

$$
d s^{2}=-\left(N^{0}\right)^{2} d t^{2}+\frac{d r^{2}}{f^{2}}+H^{2}\left(N^{\varphi} d t+d \varphi\right)^{2},
$$

where $N^{0}(r)$ is the lapse function, $N^{\varphi}(r)$ is the shift function, $f(r)$ and $H(r)$ are some functions of $r$.

Then, the Hamiltonian form of action (11) can be written as:

$$
\begin{aligned}
S= & -\Delta t \int d r N\left[\frac{2 \Pi^{2}}{H^{3}} e^{-2 \phi}-4 f^{2}\left(H \phi_{, r} e^{-2 \phi}\right)_{, r}-2 H \phi_{, r}\left(f^{2}\right)_{, r} e^{-2 \phi}\right. \\
& \left.+2 f\left(f H_{, r}\right)_{, r} e^{-2 \phi}+4 \omega H f^{2}\left(\phi_{, r}\right)^{2} e^{-2 \phi}-4 \lambda^{2} H e^{-2 \phi}\right] \\
& +\Delta t \int d r N^{\varphi}\left[2 \Pi e^{-2 \phi}\right]_{, r}+B
\end{aligned}
$$

where $\Pi^{r \varphi}=-\frac{H N^{\varphi}, r}{2 N}\left(\Pi \equiv \Pi^{r}{ }_{\varphi}\right)$ is the momentum conjugate to $g_{r \varphi}, N=\frac{N^{0}}{f}$ and $B$ is a surface term.

Upon varying the action with respect to $f(r), H(r), \Pi(r)$ and $\phi(r)$ one picks up aditional surface terms, which have to be canceled by $\delta B$ (in order that Hamilton's equations are satisfied):

$$
\delta B=-\Delta t N \delta M+\Delta t N^{\varphi} \delta J
$$

where $M$ and $J$ are, respectively, the mass and the angular momentum of the black holes:

$$
\begin{aligned}
\delta M= & \left(2 H \phi_{, r}-H_{, r}\right) e^{-2 \phi} \delta f^{2}-2 H\left[\left(f^{2}\right)_{, r}+4(\omega+1) f^{2} \phi_{, r}\right] e^{-2 \phi} \delta \phi \\
& +\left(f^{2}\right)_{, r} e^{-2 \phi} \delta H+4 f^{2} H e^{-2 \phi} \delta\left(\phi_{, r}\right)-2 f^{2} e^{-2 \phi} \delta\left(H_{, r}\right), \\
\delta J= & 4 \Pi e^{-2 \phi} \delta \phi-2 e^{-2 \phi} \delta \Pi .
\end{aligned}
$$


We choose the zero point of energy in such a way that the mass and angular momentum vanish when the horizon size goes to zero. This means that the empty space is given by:

$$
d s^{2}=-a^{2} r^{2} d t^{2}+\frac{d r^{2}}{a^{2} r^{2}}+r^{2} d \varphi^{2}
$$

which asymptotically coincides with the anti-de Sitter spacetime. For $\omega=$ $-3 / 2$ the above described procedure of choosing the zero point of energy does not apply, since for any (positive) value of $b$ one still has a black hole solution. In other words, the black hole spectrum is unbounded and there is no ground state. Thus, for $\omega=-3 / 2$ the zero point of energy is choosen (arbitrarily) to correspond to the black hole solution with $b=1$.

Inserting in eqs. (15) and (16) the solutions (5), (10) and (11) one obtains for $\omega \neq-\frac{3}{2},-1$ :

$$
\begin{aligned}
M & =\left[\frac{\omega+2}{\omega+1}+\frac{2 \omega+3}{\omega+1} \frac{\theta^{2}}{a^{2}}\right] b, \\
J & =\frac{2 \omega+3}{\omega+1} \sqrt{1+\frac{\theta^{2}}{a^{2}}} \frac{\theta}{a^{2}} b,
\end{aligned}
$$

and for $\omega=-\frac{3}{2}$ :

$$
\begin{aligned}
M & =-4 \lambda^{2}\left[1+\ln (b)\left(1-\frac{2 \theta^{2}}{\lambda^{2}}\right)-\sqrt{1-\frac{4 \theta^{2}}{\lambda^{2}} \ln (b)}\right], \\
J & =0 .
\end{aligned}
$$

Let us now analyse the solutions obtained for $\omega \neq-\frac{3}{2},-1$. We first consider the solutions with positive mass, $M>0$. Using eqs. (18) and (19), the metric (10) can be put in the form:

$$
\begin{aligned}
d s^{2}= & -\left[a^{2} r^{2}-\frac{\omega+1}{2(\omega+2)} \frac{M(1+\Omega)}{(a r)^{\frac{1}{\omega+1}}}\right] d t^{2}-\frac{\omega+1}{2 \omega+3} \frac{J}{(a r)^{\frac{1}{\omega+1}}} 2 d \varphi d t \\
+ & \frac{d r^{2}}{a^{2} r^{2}-\frac{1}{2(\omega+2)} \frac{M[(2 \omega+3) \Omega-1]}{(a r)^{\frac{1}{\omega+1}}}}+\frac{1}{a^{2}}\left[a^{2} r^{2}+\frac{M(1-\Omega)}{2(a r)^{\frac{1}{\omega+1}}}\right] d \varphi^{2}, \\
\omega & \neq-2,-\frac{3}{2},-1,
\end{aligned}
$$




$$
\begin{aligned}
d s^{2}= & -\left(a^{2} r^{2}-\frac{a^{2} J^{2}}{M} a r\right) d t^{2}-J a r 2 d \varphi d t+\frac{d r^{2}}{a^{2} r^{2}-M\left(\frac{a^{2} J^{2}}{M^{2}}-1\right) a r} \\
& +\frac{1}{a^{2}}\left(a^{2} r^{2}+M a r\right) d \varphi^{2}, \quad \omega=-2 .
\end{aligned}
$$

where we have introduced the notation:

$$
\Omega=\sqrt{1-\frac{4(\omega+1)(\omega+2)}{(2 \omega+3)^{2}} \frac{a^{2} J^{2}}{M^{2}}} .
$$

The condition that $\Omega$ remains real imposes for $-\infty<\omega<-2$ and $-1<$ $\omega<+\infty$ a restrition on the allowed values of the angular momentum:

$$
|a J| \leq \frac{|2 \omega+3| M}{2 \sqrt{(\omega+1)(\omega+2)}}
$$

The metric components become singular at $r=0$. An inspection of the Ricci and Kretschmann scalars,

$$
\begin{aligned}
& R=-6 a^{2}-\frac{\omega}{(\omega+1)^{2}} \frac{b a^{2}}{(a r)^{\frac{2 \omega+3}{\omega+1}}}, \\
& R_{a b c d} R^{a b c d}=12 a^{4}+\frac{4 \omega}{(\omega+1)^{2}} \frac{b a^{4}}{(a r)^{\frac{2 \omega+3}{\omega+1}}}+\frac{3 \omega^{2}+8 \omega+6}{(\omega+1)^{4}} \frac{b^{2} a^{4}}{(a r)^{\frac{4 \omega+6}{\omega+1}}}
\end{aligned}
$$

where $b$ is given by:

$$
\begin{array}{ll}
b=M \frac{(2 \omega+3) \Omega-1}{2(\omega+2)}, & \omega \neq-2,-\frac{3}{2},-1, \\
b=M\left(\frac{a^{2} J^{2}}{M^{2}}-1\right), & \omega=-2,
\end{array}
$$

shows that $r=0$ is a curvature singularity for $-\frac{3}{2}>\omega>-1$. For $-\frac{3}{2}<\omega<$ -1 the curvature singularity is located at $r=+\infty$ and the surface $r=0$ corresponds rather to a topological singularity.

Depending on the values of the parameter $\omega$ and the relation between the mass and the angular momentum, the metric components can also become singular for another value of the coordinate $r$, namely $a r=b^{\frac{\omega+1}{2 \omega+3}}$, which 
corresponds to the horizon. For $-\infty<\omega<-2$ there is always an horizon (for the allowed values of $J$ given by eq. (25)), while for $-2<\omega<-\frac{3}{2}$ such surface never exists, i.e., there are no black hole solutions with positive mass for this range of values of $\omega$. For $-1<\omega<+\infty$ the situation is similar to the one occurring for the four-dimensional spinning black hole, namely, the horizon exists for $|a J|<M$. Note, however, that the extreme case $|a J|=M$ is not anymore a black hole, but rather a naked singularity. A rather exotic behaviour occurs for $\omega=-2$ and $-\frac{3}{2}<\omega<-1$; for these values of $\omega$ the black hole has to spin very fast, $|a J|>M$; as soon as the angular momentum decreases, $|a J| \leq M$, the solution changes character and becomes a naked singularity. For $-\frac{3}{2}<\omega<-1$ there is a topological singularity at $r=0$. For this reason the spacetime manifold cannot be extended for negative values of $r$, contrary to the two-dimensional case [21].

Table 1 gives a summary of the conditions imposed on the angular momentum of the positive mass black holes.

For $\omega=\infty$ and $|a J|<M$, contrarily to cases mentioned above, the solution admits two horizons, given by $a r_{ \pm}= \pm \sqrt{M \Omega}$. Indeed, for $r=0$ there are no curvature or topological singularities and thus spacetime can be extended for negative values of $r$. By performing the coordinate transformation $r \rightarrow \bar{r}$, where $\bar{r}$ is given by $\bar{r}^{2}=r^{2}+\frac{M(1-\Omega)}{2 a^{2}}$, one obtains the usual form of the BTZ black hole metric with outer and inner horizons. This solution was extensively analysed in refs. [22, 60. The Penrose diagram can be infinitely extended, similary to the Penrose diagram of the four-dimensional Kerr black hole. The surface $\bar{r}=0$ is timelike and corresponds to a singularity in the causal structure and thus the solution cannot be extended for negative values of $\bar{r}$. Here, contrary to the case $\omega \neq \infty$, there is an extreme black hole, obtained for $|a J|=M$. For $J=0$, the solution admits only one horizon and the causal structure is similar to the one exhibited by the black hole solutions corresponding to $-\infty<\omega<-2$ and $-1<\omega<+\infty$, with the exception that the spacetime surface $\bar{r}=0$ is a Taub-NUT type singularity in the manifold, rather than a curvature singularity.

For $M>0$ the $g_{\varphi \varphi}$ component of the metrics (22) and (23) is always positive, which implies that there are no closed timelike curves.

We have been considering only solutions with positive mass, but in fact there is no reason to exclude the solutions with negative mass from the physical spectrum, since they do not correspond to naked singularities, but rather to black holes. An analysis identical to the one carried above for the 
positive mass black holes can also be performed. In table 1 we summarize the conditions that angular momentum must obey in order that the negative mass solutions correspond to black holes. We also remark that these solutions admit closed timelike curves. Indeed, the coordinate $\varphi$ becomes timelike in a certain region of spacetime. Since this coordinate is periodic one concludes that there are closed timelike curves in this region of spacetime.

Let us now turn to the solution obtained for $\omega=-\frac{3}{2}$ (see eq. (11)).

The stricking feature of this solution is that both surfaces $r=0$ and $r=$ $\infty$ correspond to a curvature singularity. Indeed, the Ricci and Kretschmann scalars,

$$
\begin{aligned}
& R=4 \lambda^{2}[5+6 \ln (b r)] \\
& R_{a b c d} R^{a b c d}=16 \lambda^{2}\left[11+20 \ln (b r)+12 \ln ^{2}(b r)\right]
\end{aligned}
$$

diverge for $r=0$ and $r=\infty$.

The condition that $M$ remains real imposes that the constant $b$ has to be less or equal to $e^{\frac{\lambda^{2}}{4 \theta^{2}}}$ (see eq. (20)). For any value of the constant $b$ in the interval $0<b \leq e^{\frac{\lambda^{2}}{4 \theta^{2}}}$, the solution has an horizon, given by $b r=1$. The zero point of energy was chosen (see above), so that the black hole has zero mass for $b=1$. This choice of the ground state divides arbitrarily the mass spectrum in states with positive and negative masses. Note that although there is dragging of inertial frames, the angular momentum of the solution is always zero (see eq. (21)). This kind of behaviour is unexpected for a stationary solution. The $g_{\varphi \varphi}$ component of the metrics (11) becomes negative for $r<r_{C T C}=\frac{1}{b} \exp \left\{-\frac{\lambda^{2}}{4 \theta^{2}}\right\}$. Thus, there are closed timelike curves in the region $0<r<r_{C T C}$.

The solution for $\omega=-\frac{3}{2}$ is thus a non-spinning black hole with closed timelike curves.

The Penrose diagrams of the stationary black hole solutions described above are identical to the Penrose diagrams of the static black holes (see ref. [14]), so they will not be reproduced here.

\section{Acknowledgments:}

This work was supported in part by Junta Nacional de Investigação Cientifica e Tecnológica, Portugal. 


\section{References}

[1] M. Bañados, C. Teitelboim and J. Zanelli, Phys. Rev. Lett. 69 (1992) 1849.

[2] R. B. Mann and S. F. Ross, Phys. Rev. D 47 (1993) 3319.

[3] J. D. Brown, J. Creighton and R. B. Mann, Phys. Rev. D 50 (1994) 6394.

[4] N. Cruz and J. Zanelli, Class. Quantum Grav. 12 (1995) 975.

[5] J. P. S. Lemos and V. T. Zanchin, Phys. Rev. D 53 (1996) 4684.

[6] S. Carlip, Class. Quantum Grav. 12 (1995) 2853.

[7] R. B. Mann, "Lower Dimensional Black Holes: Inside and Out", preprint WATPHYS-TH-95-02, gr-qc/9501038.

[8] G. T. Horowitz and D. L. Welch, Phys. Rev. Lett. 71 (1993) 328.

[9] N. Kaloper, Phys. Rev. D 48 (1993) 2598.

[10] J. Horne and G. T. Horowitz, Nucl. Phys. B 368 (1992) 444.

[11] G. Mandal, A. M. Sengupta and S. R. Wadia, Mod. Phys. Lett. A 6 (1991) 1685.

[12] E. Witten, Phys. Rev. D 44 (1991) 314.

[13] K. C. K. Chan and R. B. Mann, Phys. Rev. D 50 (1994) 6385; erratum, Phys. Rev. D 52 (1995) 2600.

[14] P. M. Sá, A. Kleber and J. P. S. Lemos, Class. Quantum Grav. 13 (1996) 125.

[15] M. Cadoni, "The Dualities of 3D Dilaton Gravity", INFNCA-TH9610 preprint, gr-qc/9606048.

[16] J. Stachel, Phys. Rev. D 26 (1982) 1281.

[17] J. P. S. Lemos, Phys. Lett. B 353 (1995) 46. 
[18] J. P. S. Lemos and V. T. Zanchin, "Rotating Charged Black String and Three-Dimensional Black Holes", DAF-ON preprint (Rio de Janeiro), hep-th/9511188.

[19] K. C. K. Chan and R. B. Mann, Phys. Lett. B 371 (1996) 199.

[20] T. Regge and C. Teitelboim, Ann. Phys. (NY) 88 (1974) 286.

[21] J. P. S. Lemos and P. M. Sá, Phys. Rev. D 49 (1994) 2897.

[22] M. Bañados, M. Henneaux, C. Teitelboim and J. Zanelli, Phys. Rev. D 48 (1993) 1506. 


\begin{tabular}{|l|l|l|}
\hline Range of $\omega$ & Black holes with $M>0$ & Black holes with $M<0$ \\
\hline \hline$-\infty<\omega<-2$ & $|a J| \leq \frac{|2 \omega+3| M}{2 \sqrt{(\omega+1)(\omega+2)}}$ & never \\
\hline$\omega=-2$ & $|a J|>M$ & $|a J|<|M|$ \\
\hline$-2<\omega<-\frac{3}{2}$ & never & always \\
\hline$\omega=-\frac{3}{2}$ & $J=0$ & $J=0$ \\
\hline$-\frac{3}{2}<\omega<-1$ & $|a J|>M$ & $|a J|<|M|$ \\
\hline$-1<\omega<+\infty$ & $|a J|<M$ & $|M|<|a J| \leq \frac{(2 \omega+3)|M|}{2 \sqrt{(\omega+1)(\omega+2)}}$ \\
\hline
\end{tabular}

\section{Table Caption}

\section{Table 1.}

Values of the angular momentum for which the black holes have positive and negative masses. 